\title{
Анализ распределения глубинной электропроводности в пределах Воронежского кристаллического массива по методике У. Шмукера
}

\author{
(C2020 В. Н. Груздев ${ }^{凶}$ \\ Воронежский государственный университет, \\ Университетская пл. 1, 394018, Воронеж, Российская Федерация
}

\begin{abstract}
Аннотация
Введение: Для получения информации о параметрах глубинных проводящих зон Воронежского кристаллического массива (ВКМ) и сопряженных структур использовались вариации геомагнитного поля. В работе рассматриваются обобщённые результаты магнитотеллурических исследований территории ВКМ, обработанные по методике У. Шмукера.

Методика: Обработка экспериментального материала, основанная на анализе геомагнитных вариаций в частотной области, включала расчет комплексных передаточных функций по методике У. Шмукера.

Pезультаты: По полученным данным построены карты векторов индукции и возмущения для Т = 1800 c. Приведен анализ структуры геомагнитного поля в пределах различных блоков Воронежского кристаллического массива. К северу от г. Тамбова наблюдается крупная аномалия геомагнитных вариаций, получившая название как Тамбовская аномалия электропроводности. К сочленению Белгородско-Михайловского и Крупецкого макроблоков в пределах мегаблока КМА приурочена Обоянская аномалия, характеризующая характер глубинной электропроводности.

Заключение: В пределах ВКМ выявлены аномалии электропроводности на различных уровнях земной коры, которые обусловлены объектами разной природы. В пределах Обоянской и Орловской аномалий, обусловленных графитизированными сланцами с флюидами, отмечены зоны чередования знакопеременных плотностных аномалий в верхней коре. Они формируют области аномальных напряжений в осадочном чехле над зонами пониженной плотности кристаллического фундамента с вероятным размещением в них месторождений углеводородов.

Ключевые слова: Воронежский кристаллический массив, глубинная электропроводность, комплексные передаточные функции, энергетический спектр, вектора индукции и возмущения.

Источник финансирования: Исследование выполнено при финансовой поддержке РФФИ в рамках научного проекта № 19-05-00336.

Для ичитирования: Груздев В.Н. Анализ распределения глубинной электропроводности в пределах Воронежского кристаллического массива по методике У. Шмукера // Вестник Воронежского государственного университета. Серия: Геология. 2020. №1. С. 100-108. DOI: https://doi.org/10.17308/geology.2020.1/2518
\end{abstract}

\section{Введение}

Для изучения глубинного строения Воронежского кристаллического массива (ВКМ) и сопряженных структур применялись методы магнитовариационного профилирования (МВП) и магнитотеллурического зондирования (МТ3), основанные на использовании вариаций естественного электромагнитного поля Земли.

Контент доступен под лицензией Creative Commons Attribution 4.0 License.

\footnotetext{
$\bowtie$ Груздев Владислав Николаевич, E-mail: grumerr@rambler.ru
} 


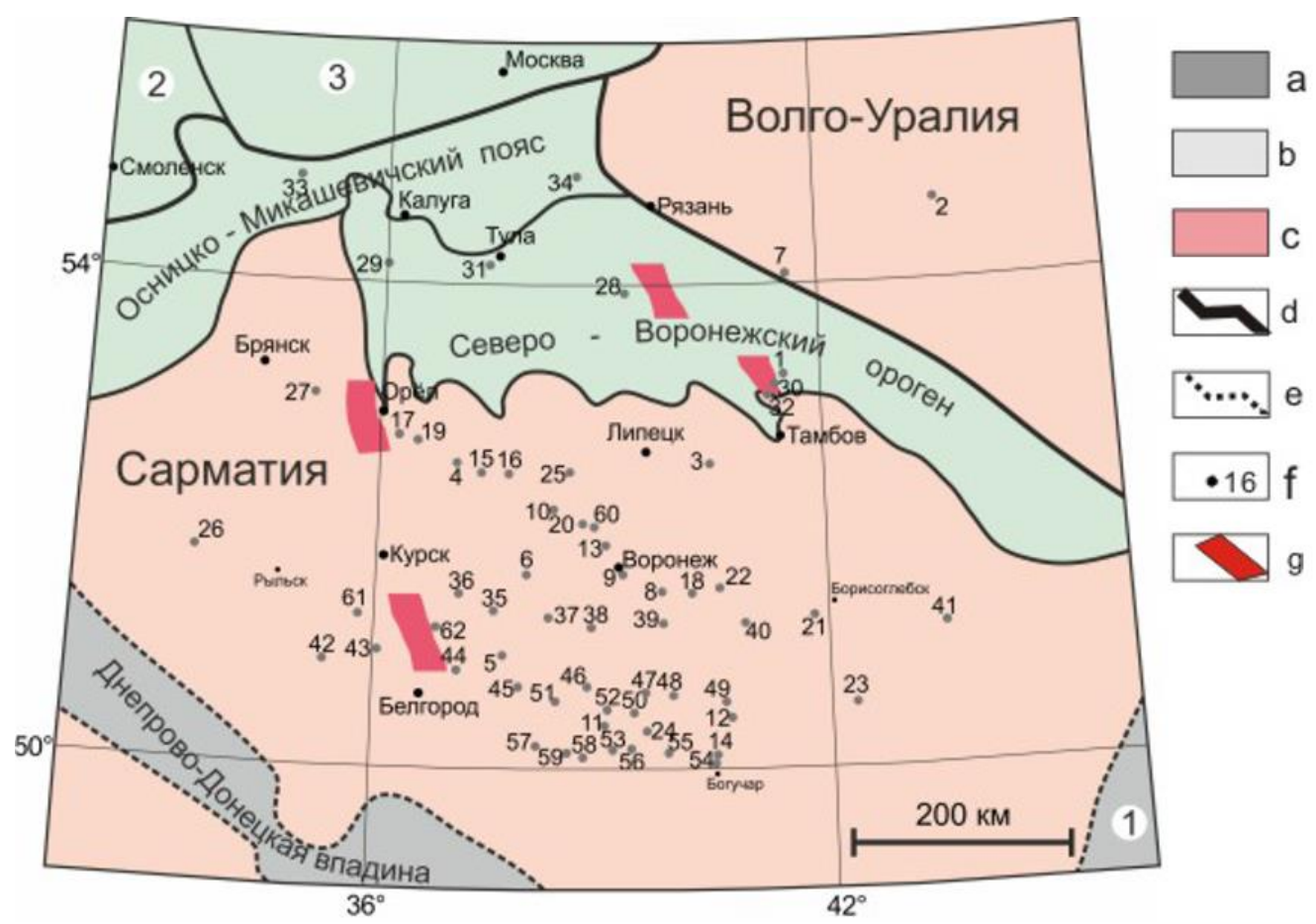

Рис. 1. Тектоника ВЕП и данные МВП. Условные обозначения: a - неопротерозой-фанерозойские структуры; $b$ - палеопротерозойские структуры; с - архейские структуры; d - границы палеоконтинентов и внутриконтинентальных орогенов; е - границы неопротерозой-фанерозойских впадин; $\mathrm{f}$ - пункты наблюдений МВП и их номера; g - зоны электропроводности. Цифры в кружках: 1 - Прикаспийская впадина, 2 - Южно-Прибалтийский и 3 - Лапландско-Среднерусский орогены.

[Fig. 1. VEP tectonics and profit center data. Legend: a - Neoproterozoic-Phanerozoic structures; b - Paleoproterozoic structures; $\mathrm{c}-$ Archean structures; $d$-boundaries of paleocontinents and intracontinental orogens; e - boundaries of the Neoproterozoic-Phanerozoic depressions; $f$ - points of observation of the profit center and their numbers; $g$ - conductivity zones. The numbers in circles are: 1 - the Caspian depression, 2 - the South Baltic and 3 - the Lapland-Central Russian orogenes.]

В методе МВП регистрация геомагнитных вариаций осуществлялась синхронно в одном базисном и нескольких рядовых пунктах, расположенных в пределах различных структур ВКМ и прилегающих районов (рис. 1). Расстояния между профилями и точками наблюдений находились в пределах от 20-30 км до 300 км и выбирались обычно с учётом простирания и положения различных структурно-тектонических блоков ВКМ, а также проводимости осадочного чехла [1] и поверхности консолидированного фундамента [2].

Геомагнитные вариации регистрировались по трём составляющим: $\mathrm{H}_{\mathrm{x}}-$ приращение поля в направлении магнитного меридиана; $\mathrm{H}_{\mathrm{y}}$ - приращение поля в направлении магнитной широты; $\mathrm{H}_{\mathrm{z}}$ - приращение поля по вертикальному направлению [3, 4].

\section{Методика}

Для получения информации о параметрах проводящих зон использовалась методика обработки экспериментального материала, основанная на анализе геомагнитных вариаций в частотной области. Вариации «бухтообразных» возмущений, зарегистрированные синхронно в базисном и в одном или нескольких рядовых пунктах, подвергались цифровой фильтрации [4].

При фильтрации трансформировались исходные временные функции $\mathrm{H}_{\mathrm{x}}(\mathrm{t}), \mathrm{H}_{\mathrm{y}}(\mathrm{t}), \mathrm{H}_{\mathrm{z}}(\mathrm{t})$ в частотную область при сохранении линейных связей между компонентами геомагнитного поля. Цифровая фильтрация магнитовариационных данных осуществлялась с помощью свёртки вида:

$$
F_{\phi}(\tau)=\int_{-\infty}^{+\infty} F(t) \cdot g(t-\tau) d t
$$

где: $F(t)$ - исходный процесс, в качестве которого выступают компоненты наблюдённого поля $\mathrm{H}_{\mathrm{x}}(\mathrm{t})$, $\mathrm{H}_{\mathrm{y}}(\mathrm{t})$ и $\mathrm{H}_{\mathrm{z}}(\mathrm{t}) ; F_{\phi}(\tau)$ - фильтрованный процесс; $\mathrm{g}(t-\tau)$ комплексная переходная характеристика цифрового фильтра. Свёртка осуществлялась с помощью переходной характеристики, представленной колоколообразной функцией:

$$
g(t-\tau)=\sqrt{\frac{\pi}{a}} \cdot \frac{1}{T} \cdot e^{-\frac{\pi^{2}}{a} \cdot\left(\frac{t-\tau}{T}\right)^{2}+i \cdot \frac{2 \pi}{T}(t-\tau)},
$$

где: $T$ - выделяемый период, $a$ - числовой безразмерный коэффициент.

Данная переходная характеристика, при величине параметра $a=9$, обеспечивает оптимальность фильтрации, так как имеет относительно высокую разрешающую способность и затухает на сравнительно коротком временном интервале. Выбранный оптимальный фильтр осуществляет преобразование 
исходного процесса $F(\mathrm{t})$ при длине реализации сигнала $\mathrm{L}=3 \mathrm{~T}$.

Смещение интегрального преобразования увеличивается из-за присутствия низкочастотного фона (региональной составляющей поля вариаций) в исходной реализации. Для повышения эффективности фильтрации, низкочастотный фон удалялся путём вычитания из наблюдённого поля региональной составляющей, полученной методом сглаживания согласно [5].

Шаг дискретизации выбирался с учётом обеспечения 10 отчётов на выделяемый период полезного сигнала. Для получения фильтрованного процесса с периодом 1800 с оцифровка проводилась с шагом 180 с. За середину реализации обычно принималась абсцисса, соответствующая экстремальным значениям $\mathrm{H}_{\mathrm{z}}-$ компонента. Колебания с периодом меньшим удвоенного шага дискретизации усреднялись. Цифровая фильтрация выполнялась при $\tau=0$.

Для последующего анализа использовались мгновенные значения фильтрованных вариаций компонент геомагнитного поля в комплексной области, соответствующие экстремальному значению $\mathrm{H}_{\mathrm{z}}-$ компонента. По совокупности исходных реализаций $\mathrm{H}_{\mathrm{x}}(\mathrm{t})$, $\mathrm{H}_{\mathrm{y}}(\mathrm{t}), \mathrm{H}_{\mathrm{z}}(\mathrm{t})$, соответствующих одному импульсу геомагнитной вариации, получались мгновенные значения вещественных и мнимых величин: $\mathrm{Re} \mathrm{H}_{\mathrm{x}}, \mathrm{Jm}$

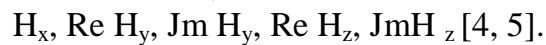

Цифровая фильтрация выполнялась по синхронным реализациям на базисном и рядовом пунктах наблюдения. По каждому пункту наблюдения фильтрация проводилась для периода вариаций Т = 1800 с. с использованием 7-9 реализаций.

Максимальные мгновенные комплексные амплитуды фильтрованных компонент поля применялись для расчёта комплексных передаточных функций по методике У. Шмукера, которая основана на статистической корреляции аномальных и нормальных частей поля. Для каждой точки наблюдения определялась матрица комплексных передаточных функций. Элементы последней позволяют дать качественную характеристику латеральным геоэлектрическим неоднородностям в недрах Земли. Получение устойчивых комплексных передаточных функций обусловлено использованием для корреляции аномальных и нормальных частей поля их энергетические и взаимно-энергетические спектры, рассчитанные по комплексным мгновенным значениям фильтрованных процессов [6].

Для упрощения записей введём обозначения: $\mathrm{H}=$ $\mathrm{H}_{\mathrm{x}}(\mathrm{t}) ; \mathrm{D}=\mathrm{H}_{\mathrm{y}}(\mathrm{t}) ; \mathrm{Z}=\mathrm{H}_{\mathrm{z}}(\mathrm{t})$. Тогда: $\mathrm{S}_{\mathrm{H}}=\frac{C_{H} \cdot C_{H}^{*}}{T_{0}} ; \mathrm{S}_{\mathrm{ZH}}=$ $\mathrm{S}_{\mathrm{HZ}}^{*}=\frac{C_{Z} \cdot C_{H}^{*}}{T_{0}}$, где $\mathrm{C}_{\mathrm{H}}$ и $\mathrm{C}_{\mathrm{Z}}-$ комплексные коэффициенты гармонического анализа исходных реализаций $\mathrm{H}_{\mathrm{x}}(t)$ и $\mathrm{H}_{\mathrm{z}}(t), \mathrm{C}_{\mathrm{H}}^{*}-$ число комплексносопряженное к $\mathrm{C}_{\mathrm{H}}, \mathrm{S}_{\mathrm{H}}-$ энергетический спектр (индекс внизу обозначает компонент поля), $\mathrm{S}_{\mathrm{ZH}}-$ взаимно-энергетический спектр (индекс внизу обозначает компоненты поля, между которыми рассчитывается спектр), $\mathrm{T}_{0}$ - длина реализации.

Для корреляции энергетических спектров между аномальными и нормальными полями, последние выделялись из наблюдённых полей следующим образом: в качестве нормального поля вариаций горизонтальных компонент, приняты те их значения, которые наблюдаются вдали от аномальных зон, а нормальное поле вертикального компонента принято за 0. Так как точки наблюдения расположены в средних широтах и на расстоянии не более 380 км от базисного пункта, то с погрешностью до $12 \%$ градиентами горизонтальных составляющих нормального поля можно пренебречь [6].

Пункт наблюдения 60 (рис. 1), находящийся вдали от аномальных зон, был условно принят за базисный, и вариации горизонтальных компонент поля в нем $\left(\mathrm{H}_{0}, \mathrm{D}_{0}\right)$ считаются нормальными. Аномальные части $\mathrm{H}$ и D - компонент в текущей $i$-ой точке определяются в виде: $\mathrm{H}_{a i}=\mathrm{H}_{i}-\mathrm{H}_{0}, \mathrm{D}_{a i}=\mathrm{D}_{i}-\mathrm{D}_{0}$. Аномальная часть $\mathrm{Z}$ - компонента в $i$-ой точке определяется в виде: $\mathrm{Z}_{a i}=$ $\mathrm{Z}_{i}$, где $\mathrm{H}_{i}, \mathrm{D}_{i}, \mathrm{Z}_{i}$ - комплексные мгновенные значения фильтрованных, соответственно, $\mathrm{H}_{\mathrm{x}}, \mathrm{H}_{\mathrm{y}}$ и $\mathrm{H}_{\mathrm{z}}-$ компонент поля.

По комплексным мгновенным значениям фильтрованных компонент нормальных и аномальных полей для каждого пункта наблюдения рассчитывались энергетические $\left(\mathrm{S}_{\mathrm{Ho}}, \mathrm{S}_{\mathrm{Do}}\right)$ и взаимно - энергетические $\left(\mathrm{S}_{\mathrm{ZaHo}}, \mathrm{S}_{\mathrm{ZaDo}}, \mathrm{S}_{\mathrm{HaHo}}, \mathrm{S}_{\mathrm{HaDo}}, \mathrm{S}_{\mathrm{DaHo}}, \mathrm{S}_{\text {DaDo }}, \mathrm{S}_{\mathrm{HoDo}}, \mathrm{S}_{\text {DoHo }}\right)$ спектры, которые образуют линейные связи вида:

$$
\begin{aligned}
& \mathrm{S}_{\mathrm{ZaHo}}=\mathrm{Z}_{\mathrm{H}} \cdot \mathrm{S}_{\mathrm{Ho}}+\mathrm{z}_{\mathrm{D}} \cdot \mathrm{S}_{\mathrm{DoHo}}+\mathrm{z}_{\mathrm{Z}} \cdot \mathrm{S}_{\mathrm{ZoHo}}, \mathrm{S}_{\mathrm{ZaDo}}=\mathrm{z}_{\mathrm{H}} \cdot \mathrm{S}_{\mathrm{HoDo}}+\mathrm{z}_{\mathrm{D}} \cdot \mathrm{S}_{\mathrm{Do}}+\mathrm{z}_{\mathrm{Z}} \cdot \mathrm{S}_{\mathrm{ZoDo}} \text {, } \\
& \mathrm{S}_{\mathrm{ZaZo}}=\mathrm{Z}_{\mathrm{H}} \cdot \mathrm{S}_{\mathrm{HoZo}}+\mathrm{Z}_{\mathrm{D}} \cdot \mathrm{S}_{\mathrm{DoZo}}+\mathrm{z}_{\mathrm{Z}} \cdot \mathrm{S}_{\mathrm{Zo}}, \mathrm{S}_{\mathrm{HaHo}}=\mathrm{h}_{\mathrm{H}} \cdot \mathrm{S}_{\mathrm{Ho}}+\mathrm{h}_{\mathrm{D}} \cdot \mathrm{S}_{\mathrm{DoHo}}+\mathrm{h}_{\mathrm{Z}} \cdot \mathrm{S}_{\mathrm{ZoHo}} \text {, } \\
& \mathrm{S}_{\mathrm{HaDo}}=\mathrm{h}_{\mathrm{H}} \cdot \mathrm{S}_{\mathrm{HoDo}}+\mathrm{h}_{\mathrm{D}} \cdot \mathrm{S}_{\mathrm{Do}}+\mathrm{h}_{\mathrm{Z}} \cdot \mathrm{S}_{\mathrm{ZoDo}}, \mathrm{S}_{\mathrm{HaZo}}=\mathrm{h}_{\mathrm{H}} \cdot \mathrm{S}_{\mathrm{HoZ}}+\mathrm{h}_{\mathrm{D}} \cdot \mathrm{S}_{\text {DoZo }}+\mathrm{h}_{\mathrm{Z}} \cdot \mathrm{S}_{\mathrm{zo}} \text {, } \\
& \mathrm{S}_{\mathrm{DaHo}}=\mathrm{d}_{\mathrm{H}} \cdot \mathrm{S}_{\mathrm{Ho}}+\mathrm{d}_{\mathrm{D}} \cdot \mathrm{S}_{\mathrm{DoHo}}+\mathrm{d}_{\mathrm{Z}} \cdot \mathrm{S}_{\mathrm{ZoHo}}, \mathrm{S}_{\mathrm{DaDo}}=\mathrm{d}_{\mathrm{H}} \cdot \mathrm{S}_{\mathrm{HoDo}}+\mathrm{d}_{\mathrm{D}} \cdot \mathrm{S}_{\mathrm{Do}}+\mathrm{d}_{\mathrm{Z}} \cdot \mathrm{S}_{\mathrm{ZoDo}} \text {, } \\
& \mathrm{S}_{\text {DaZo }}=\mathrm{d}_{\mathrm{H}} \cdot \mathrm{S}_{\mathrm{HoZ}}+\mathrm{d}_{\mathrm{D}} \cdot \mathrm{S}_{\text {DoZo }}+\mathrm{d}_{\mathrm{Z}} \cdot \mathrm{S}_{\mathrm{Zo}} \text {, }
\end{aligned}
$$

где: $\mathrm{z}_{\mathrm{H}}, \mathrm{z}_{\mathrm{D}}, \mathrm{z}_{\mathrm{Z}}, \mathrm{h}_{\mathrm{H}}, \mathrm{h}_{\mathrm{D}}, \mathrm{h}_{\mathrm{Z}}, \mathrm{d}_{\mathrm{H}}, \mathrm{d}_{\mathrm{D}}, \mathrm{d}_{\mathrm{Z}}$ - передаточные функции.

Учитывая характер поведения нормального поля в средних широтах, т.е. $Z_{0}=0$, члены, содержащие взаимно - энергетические спектры $\left(\mathrm{S}_{\mathrm{ZoHo}}, \mathrm{S}_{\mathrm{ZoDo}}, \mathrm{S}_{\mathrm{Zo}}\right.$, $\left.\mathrm{S}_{\mathrm{ZaZo}}, \mathrm{S}_{\mathrm{ZaHo}}, \mathrm{S}_{\mathrm{ZaDo}}\right)$ в уравнениях, равны нулю. $\mathrm{B}$ результате получим соотношения для вычисления передаточных функций:

$$
\begin{aligned}
& z_{H}=\frac{S_{Z_{a} H_{o}} \cdot S_{D_{o}}-S_{Z_{a} D_{o}} \cdot S_{D_{o} H_{o}}}{S_{H_{o}} \cdot S_{D_{o}}-\left(S_{H_{o} D_{o}}\right)^{2}}, \\
& z_{D}=\frac{S_{Z_{a} D_{o}} \cdot S_{H_{o}}-S_{Z_{a} H_{o}} \cdot S_{D_{o} H_{o}}}{S_{H_{o}} \cdot S_{D_{o}}-\left(S_{H_{o} D_{o}}\right)^{2}},
\end{aligned}
$$




$$
\begin{aligned}
& h_{H}=\frac{S_{H_{a} H_{o}} \cdot S_{D_{o}}-S_{H_{a} D_{o}} \cdot S_{D_{o} H_{o}}}{S_{H_{o}} \cdot S_{D_{o}}-\left(S_{H_{o} D_{o}}\right)^{2}}, \\
& h_{D}=\frac{S_{H_{a} D_{o}} \cdot S_{H_{o}}-S_{H_{a} H_{o}} \cdot S_{D_{o} H_{o}}}{S_{H_{o}} \cdot S_{D_{o}}-\left(S_{H_{o} D_{o}}\right)^{2}}, \\
& d_{H}=\frac{S_{D_{a} H_{o}} \cdot S_{D_{o}}-S_{D_{a} D_{o}} \cdot S_{D_{o} H_{o}}}{S_{H_{o}} \cdot S_{D_{o}}-\left(S_{H_{o} D_{o}}\right)^{2}}, \\
& d_{D}=\frac{S_{D_{a} D_{o}} \cdot S_{H_{o}}-S_{D_{a} H_{o}} \cdot S_{D_{o} H_{o}}}{S_{H_{o}} \cdot S_{D_{o}}-\left(S_{H_{o} D_{o}}\right)^{2}} .
\end{aligned}
$$

Наблюдаемые зависимости $\mathrm{H}_{\mathrm{x}}(t), \mathrm{H}_{\mathrm{y}}(t), \mathrm{H}_{\mathrm{z}}(t)$ содержат определённую погрешность, следовательно, эта погрешность будет существовать и при расчёте комплексных передаточных функций. Для характеристики качества расчёта передаточных функций (статистической оценки корреляции между аномальными и нормальными полями) определяются остаточные погрешности в виде:

$$
\begin{aligned}
& \varepsilon_{Z}=\left(1-\frac{z_{H} \cdot S_{H_{o} Z_{a}}+z_{D} \cdot S_{D_{o} Z_{a}}}{S_{Z_{a}}}\right)^{1 / 2}, \\
& \varepsilon_{\mathrm{h}}=\left(1-\frac{h_{H} \cdot S_{H_{o} H_{a}}+h_{D} \cdot S_{D_{o} H_{a}}}{S_{H_{a}}}\right)^{1 / 2}, \\
& \varepsilon_{\mathrm{d}}=\left(1-\frac{d_{H} \cdot S_{H_{o} D_{a}}+d_{D} \cdot S_{D_{o} D_{a}}}{S_{D_{a}}}\right)^{1 / 2} .
\end{aligned}
$$

Значения остаточных погрешностей используются для вычисления когерентности между аномальными и нормальными полями: $\mathrm{Co}_{Z}=\sqrt{1-\varepsilon_{Z}^{2}}, \quad \mathrm{Co}_{h}=\sqrt{1-\varepsilon_{h}^{2}}$, $C o_{d}=\sqrt{1-\varepsilon_{d}^{2}}$. Чем меньше $\varepsilon(0 \leq \varepsilon \leq 1)$, или чем больше $C o(0 \leq \mathrm{Co} \leq 1)$, тем лучше корреляция между аномальными и нормальными полями. Верхний допустимый предел $\varepsilon$ определяется из выражения: $\sqrt{\frac{N-4}{N-2}}$, где $N-$ число используемых реализаций поля геомагнитных вариаций.

Для графической иллюстрации комплексных передаточных функций $z_{H}$ и $z_{D}$ использовались стрелки индукции или вектора индукции Шмукера, определяемые выражениями вида:

$$
\begin{aligned}
\vec{C}_{U} & =-z_{H}(u) \cdot \vec{i}-z_{D}(u) \cdot \vec{j}, \\
\vec{C}_{V} & =-z_{H}(v) \cdot \vec{i}-z_{D}(v) \cdot \vec{j},
\end{aligned}
$$

где $\vec{C}_{U}$ - действительная часть вектора индукции, $\vec{C}_{V}$ - мнимая часть вектора индукции, $\vec{i}-$ единичный вектор, направленный вдоль магнитного меридиана, $\vec{j}$ - единичный вектор, направленный вдоль магнитной широты, $z_{H}(u), z_{D}(u) \quad$ действительные части передаточных функций, $z_{H}(v)$, $z_{D}(v)$ - мнимые части передаточных функций.

Для графической иллюстрации комплексных передаточных функций $h_{H}, h_{D}, d_{H}, d_{D}$ строятся стрелки возмущения или векторы возмущения Шмукера вида:

$$
\begin{gathered}
\vec{P}_{U}=h_{H}(u) \cdot \vec{i}+d_{H}(u) \cdot \vec{j}, \\
\vec{P}_{V}=h_{H}(v) \cdot \vec{i}+d_{H}(v) \cdot \vec{j}, \\
\vec{q}_{U}=h_{D}(u) \cdot \vec{i}+d_{D}(u) \cdot \vec{j}, \\
\vec{q}_{V}=h_{D}(v) \cdot \vec{i}+d_{D}(v) \cdot \vec{j},
\end{gathered}
$$

где: $\vec{P}_{U}, \vec{q}_{U}-$ действительные части векторов возмущения, $\vec{P}_{V}, \quad \vec{q}_{V}-$ мнимые части векторов возмущения, $\quad h_{H}(u), \quad h_{D}(u), \quad d_{H}(u), \quad d_{D}(u) \quad-$ действительные части передаточных функций, $h_{H}(v)$, $h_{D}(v), d_{H}(v), d_{D}(v)$ - мнимые части передаточных функций.

Степень статистической достоверности расчёта векторов индукции и возмущения графически изображается доверительной окружностью, радиус которой, например для $\mathrm{Z}_{\mathrm{H}}$ и $\mathrm{Z}_{\mathrm{D}}$ определяется в виде: $\mathrm{R}$ $=\varepsilon_{Z} \cdot \sqrt{C_{U}^{2}+C_{V}^{2}}$.

\section{Результаты}

Характер поведения действительный и мнимых векторов индукции и возмущения отражает особенности строения региона как в целом, так и в конкретной точке наблюдения [7].

Направление вектора индукции $\vec{C}_{U}$ указывает на положение зон высокой проводимости в плане. Поведение вектора $\vec{C}_{V}$ и характер его зависимости от периода свидетельствует о поверхностной или глубинной природе проводящих зон. Коллинеарность векторов $\vec{C}_{U}$ и $\vec{C}_{V}$ указывает на двумерный, а нарушение коллинеарности - на трёхмерный характер проводящих зон. Таким образом, действительный и мнимый векторы индукции дают представление о геометрии среды, природе и положении зон повышенной проводимости (рис. 2).

Векторы возмущения $\vec{P}_{U}$ и $\vec{P}_{V}$ характеризуют распределение горизонтальных компонент аномального поля, индуцированного нормальным, поляризованным вдоль магнитного меридиана полем (рис. 3).

Векторы возмущения $\vec{q}_{U}$ и $\vec{q}_{V}$ связаны с распределением горизонтальных компонент аномального поля, обусловленного нормальным полем, поляризованным вдоль магнитной широты (рис. 4). 


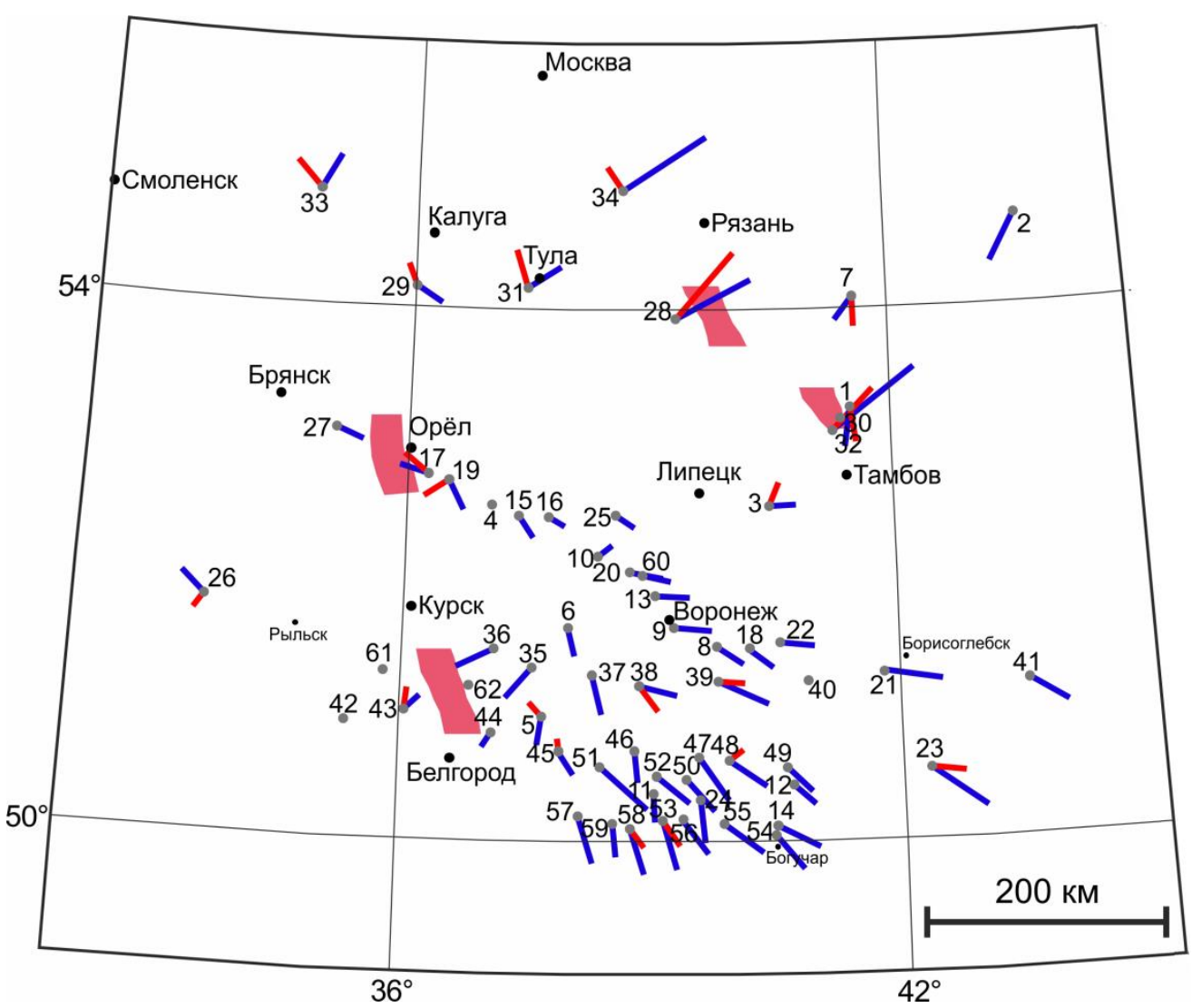

Рис. 2. Вектора индукции Шмукера ( $\vec{C}$ ) для $\mathrm{T}=1800$ с: действительная часть $\left(\vec{C}_{U}\right)$ синий цвет, мнимая часть $\left(\vec{C}_{V}\right)$ - красный цвет, - зоны электропроводности.

[Fig. 2. The Schmucker induction vector $(\vec{C})$ for $\mathrm{T}=1800 \mathrm{~s}$ : the real part ( $\vec{C}_{U}$ ) - is blue, the imaginary $\left(\vec{C}_{V}\right)-$ part is red, conductivity zones.]

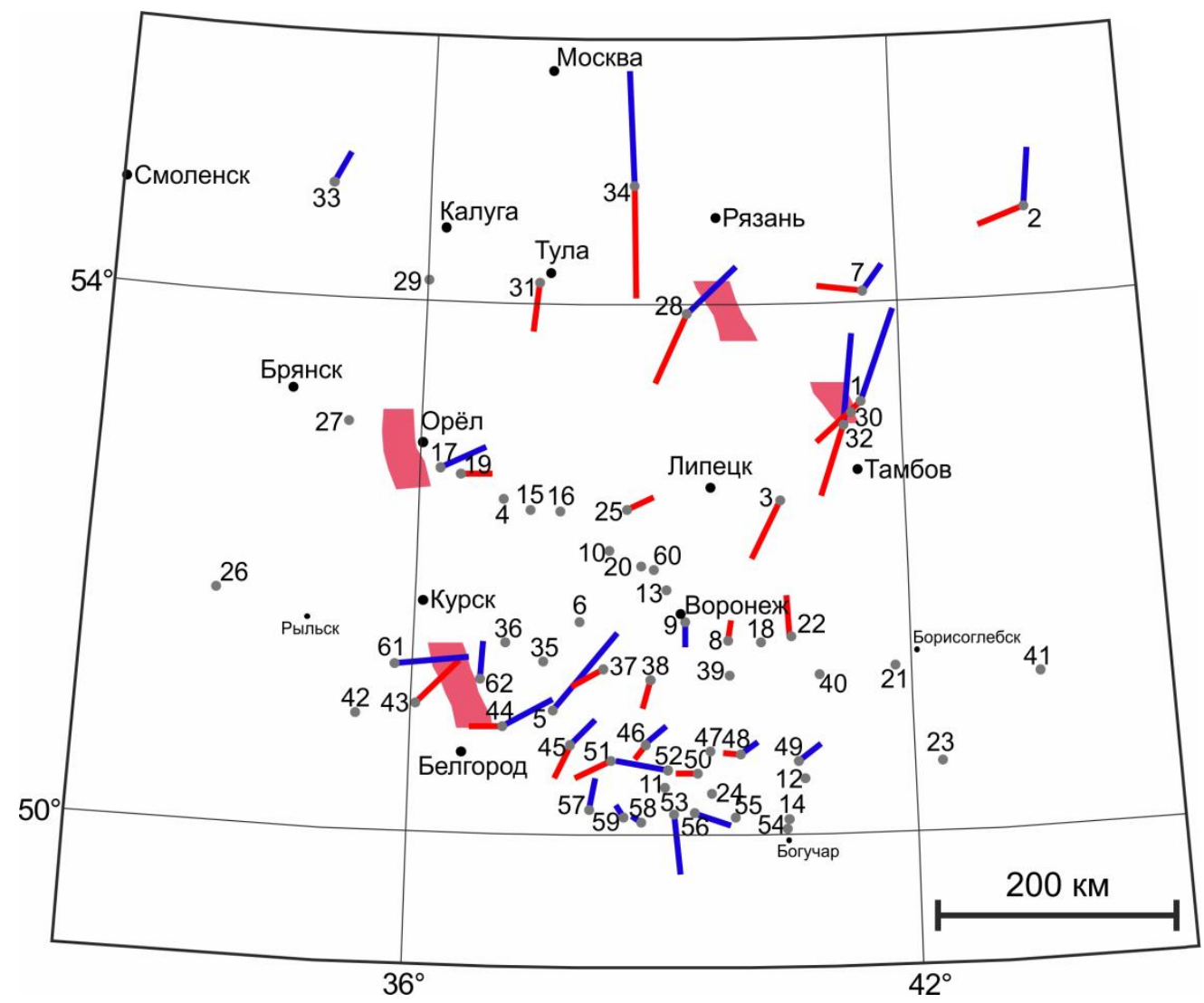

Рис. 3. Вектора возмущений Шмукера ( $\vec{P}$ ) для $\mathrm{T}=1800$ с: действительная часть $\left(\vec{P}_{U}\right)$ - синий цвет, мнимая часть $\left(\vec{P}_{V}\right)$ - красный цвет, - зоны электропроводности.

[Fig. 3. Schmucker's vector perturbations $(\vec{P})$ for $\mathrm{T}=1800 \mathrm{~s}$ : the real part $\left(\vec{P}_{U}\right)$ - blue, the imaginary part $\left(\vec{P}_{V}\right)-$ red, - conductivity zones.] 


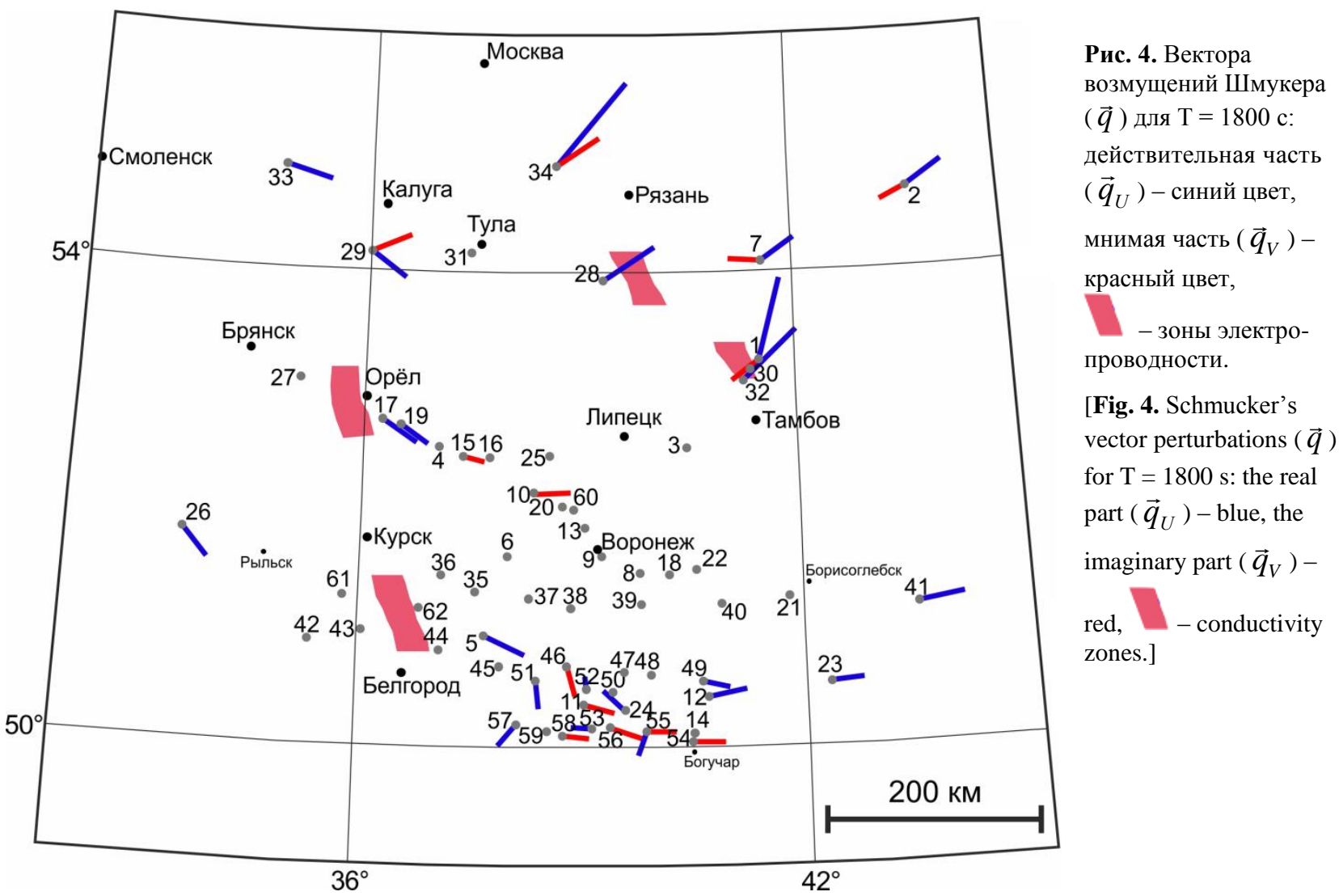

Поворот векторов $\vec{P}_{U}, \vec{P}_{V}, \vec{q}_{U}, \vec{q}_{V}$ на $90^{0}$ против часовой стрелки даёт направление аномального тока, индуцированного нормальным током, текущим, соответственно, вдоль магнитной широты и магнитного меридиана

При северо-восточной поляризации первичного поля для бухтообразных возмущений $(\mathrm{T}=1800 \mathrm{c})$ геомагнитное поле имеет наиболее аномальный характер поведения. В пределах мегаблока КМА, за исключением его юго-восточной части, отмечается региональное положительное поле в $\mathrm{H}_{\mathrm{z}}-$ и в $\mathrm{H}_{\mathrm{x}}$ компонентах. Все остальные крупные структуры ВКМ характеризуются отрицательным полем данных компонент, интенсивность которого плавно растет по модулю от свода ВКМ в сторону РСП. На фоне регионального изменения поля выделяются локальные аномальные зоны изометрической формы, которые приурочены к зонам разломов различного порядка и сочленениям структурно-тектонических блоков ВКМ.

К северу от г. Тамбова наблюдается крупная аномалия геомагнитных вариаций, получившая название как Тамбовская аномалия электропроводности. Аномальный характер поля вариаций зарегистрирован в 6 пунктах на периодах 600, 1800, 3600 и 5400 с. Наиболее четко данная геомагнитная аномалия проявляется на периоде 1800 с (рис. 2, 3, 4). Аномалия имеет квазидвумерных характер простирания в северозападном направлении на расстояние более 1000 км при ширине до 150 км. Интенсивность аномального поля в горизонтальных компонентах составляет 100\% от уровня нормального поля, а отрицательные значения амплитуды $\mathrm{H}_{\mathrm{z}}$-компонента по уровню достигают значений горизонтальных компонент нормального поля. Наиболее четко аномальная зона проявляется при поляризации горизонтального вектора в северовосточном и юго-западном направлениях.

В районе п.н. 43, 44, 61, 62 расположена четко выраженная Обоянская аномалия геомагнитных вариаций, которая приурочена к сочленению БелгородскоМихайловского и Крупецкого макроблоков в пределах мегаблока КМА, и характеризует характер глубинной электропроводности (рис. 2, 3, 4). Неколлинеарность мнимой и действительной частей векторов индукции и возмущения в 2 пунктах, а также распределение синхронных амплитуд различных компонент, указывает на трехмерный характер распределения аномального поля и соответственно параметров электропроводности. Аномалия в плане имеет размеры в поперечнике до 150 км и выделяется по четкой инверсии $\mathrm{H}_{\mathrm{z}}$-компоненты и одновременном увеличении амплитуды $\mathrm{H}_{\mathrm{x}}$-компоненты на 44\%. В общем плане интенсивность поля в аномальной зоне на $30-40 \%$ превышает нормальный фон.

\section{Заключение}

Аномалии электропроводности, выявленные в пределах ВКМ на различных уровнях земной коры, обусловлены объектами разной природы $[8,9,10]$.

Приповерхностные аномалии связаны со спецификой петрологических ассоциаций, выполняющих 
наложенные геологические структуры, выявленные по данным плотностного моделирования.

Аномалия электропроводности Тамбовская на уровне кровли гравиактивного слоя обусловлена наличием высокоминерализованного флюида в открытых порах, генетически связанного с процессами дилатансии в зонах глубинных разломов, отмечаемых на глубинных срезах плотностной модели ВКМ (рис. 5) [8, 9, 10].
В пределах аномалий электропроводности, таких как Обоянская и Орловская, обусловленных графитизированными сланцами с флюидами, отмечены зоны чередования знакопеременных плотностных аномалий в верхней коре, которые возможно формируют области аномальных напряжений в осадочном чехле над зонами пониженной плотности кристаллического фундамента с возможным размещением в них месторождений углеводородов (рис. 5).

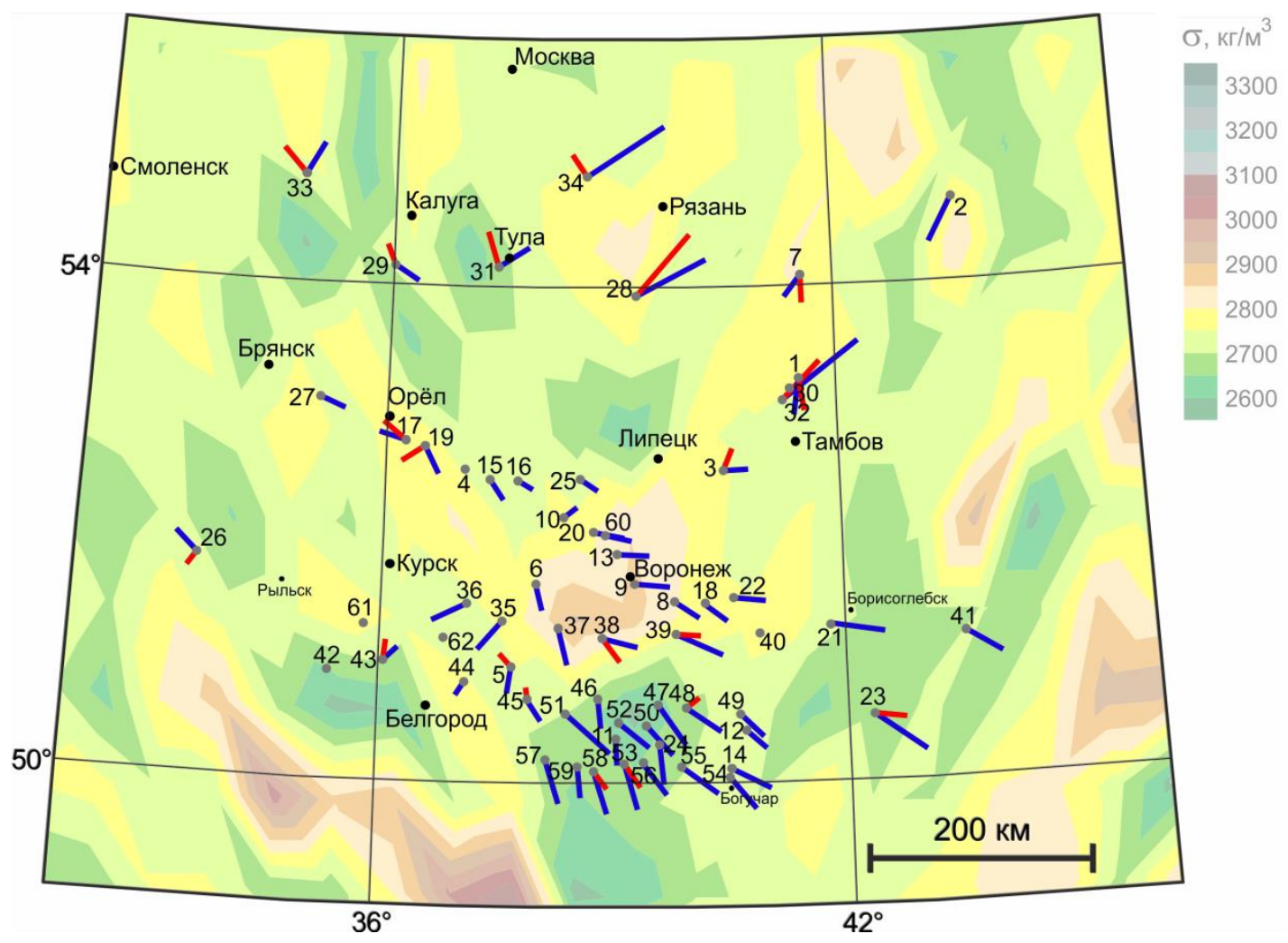

Рис. 5. Распределение плотности в литосфере на уровне кровли гравиактивного слоя и векторов индукции Шмукера ( $\vec{C}$ ) для Т $=1800$ с: действительная часть $\left(\vec{C}_{U}\right)$ - синий цвет, мнимая часть $\left(\vec{C}_{V}\right)$ - красный цвет.

[Fig. 5. The density distribution in the lithosphere at the roof level of the gravity layer and the Schmucker induction vectors ( $\vec{C}$ ) for $\mathrm{T}=1800$ s: the real part $-\left(\vec{C}_{U}\right)$ is blue, the imaginary $\left(\vec{C}_{V}\right)$ - part is red.]

Конфликт интересов: Автор декларирует отсутствие явных и потенциальных конфликтов интересов, связанных с публикацией настоящей статьи.

\section{ЛИТЕРАТУРА}

1. Жаворонкин В. И., Груздев В. Н., Фаустова Ю. С. Карта суммарной продольной электрической проводимости осадочного чехла воронежского кристаллического массива и его обрамления // Вопросы теории и практики геологической интерпретации геофизических полей. Материалы 45-й сессии Международного семинара им. Д. Г. Успенского. Казань. 2018. С. 127-128.

2. Груздев В. Н. Суммарная электропроводность консолидированной части земной коры // Вестник Воронежского государственного университета. Серия: Геология. 2014. № 2. С. 182-185.

3. Рокитянский И. И., Логвинов И. М., Максимов В. М. Площадные магнитовариационные исследования в цент- ральной части Русской платформы. // Изв. АН СССР, Физика Земли. 1977. № 9. С. 107-112.

4. Жданов М. С., Максимов В. М., Груздев В. Н. О результатах магнитовариационных исследований на территории ВКМ и сопредельных структур центра Русской платформы // Электромагнитные зондирования. Тез. докл. V11 Всес. щколы-семинара. Москва. 1984. С. 75.

5. Груздев В. Н. Геоэлектрическая модель Воронежского кристаллического массива // Вопросы теории и практики геологической интерпретации гравитационных, магнитных и электрических полей. Тез. докл. международной конференции. Воронеж. 1996. С.72-73.

6. Максимов В. М., Груздев В. Н. Использование методики Шмукера при обработке и интерпретации магнитовариационных данных // Геофизические методы поисков и разведки рудных и нерудных месторождений. Свердловск, 1986. Вып. 12. С. 38-44.

7. Груздев В. Н., Жаворонкин В. И. Коровые аномалии глубинной электропроводности Воронежского кристалли- 
ческого массива // Вестник Воронежского государственного университета. Серия: Геология. 2015. № 1. С. 94-101.

8. Муравина О. М. Плотностная модель земной коры Воронежского кристаллического массива // Вестник Воронежского государственного университета. Серия: Геология. 2016. № 1. С. 108-114.

9. Глазнев В. Н., Минц О. М., Муравина О. М. Плотностное моделирование земной коры центральной части Восточ-
но-Европейской платформы // Вестн. Краунц. Науки о Земле. Вып. 29. 2016. № 1. с. 53-63.

10. Минц М. В., Глазнев В. Н., Муравина О. М. Глубинное строение коры юго-востока Воронежского кристаллического массива по геофизическим данным: геодинамическая эволюция в палеопротерозое и современное состояние коры // Вестник Воронежского государственного университета. Серия: Геология. 2017. № 4. С. 5-23.

\title{
GEOPHYSICS
}

UDC 550.311.(470.32)

DOI: https://doi.org/10.17308/geology.2020.1/2518

ISSN 1609-0691

Received: 03.02.2020

Accepted: 20.02.2020

Published online: 25.03 .2020

\section{The analysis of the distribution of deep electrical conductivity within the Voronezh Crystalline Massif using U. Schmuker's method}

\author{
C2020 V. N. Gruzdev ${ }^{\bowtie}$ \\ Voronezh State University, 1 Universitetskaya pl., Voronezh, 394018, Russian Federation
}

\begin{abstract}
Introduction: Geomagnetic field variations were used to obtain information regarding the parameters of the deep conductive zones of the Voronezh Crystalline Massif (VCM) and conjugated structures. The study considers the generalized results of magnetotelluric research of the VCM territory processed using U. Schmucker's method.

Methodology: The processing of the experimental material based on the analysis of geomagnetic variations in the frequency domain included the calculation of complex transfer functions using U. Schmucker's method.

Results and discussion: Based on the obtained data the charts of induction and disturbance vectors for $\mathrm{T}=$ $1800 \mathrm{~s}$ were prepared. The structure of the geomagnetic field within various blocks of the Voronezh Crystalline Massif was analysed. To the north of Tambov there is a large geomagnetic variation anomaly, called the Tambov Electrical Conductivity Anomaly. At the junction of the Belgorod-Mikhailovsky and Krupetsky macroblocks within the megablock of the Kursk Magnetic Anomaly (KMA) there is the Oboyan Anomaly, which characterizes the nature of the deep electrical conductivity.

Conclusion: Electrical conductivity anomalies were detected within the VCM at various levels of the earth's crust, which are caused by objects of various natures. Within the Oboyan and Oryol Anomalies, which are caused by graphitized shales with fluid, interchange zones of alternating density anomalies were registered in the upper crust, which probably form the abnormal stress domains in the sedimentary cover above the zones of reduced density of the crystalline basement, possibly with hydrocarbon deposits in them.
\end{abstract}

Keywords: Voronezh Crystalline Massif, deep electrical conductivity, complex transit functions, energy spectrum, induction and disturbance vectors.

Funding: The study was supported by the Russian Foundation for Basic Research, project no. 19-05-00336.

\footnotetext{
$\bowtie$ Vladislav N. Gruzdev, E-mail: grumerr@rambler.ru
} 
For citation: Gruzdev V. N. The analysis of the distribution of deep electrical conductivity within the Voronezh Crystalline Massif using U. Schmuker's method. Vestnik Voronezhskogo gosudarstvennogo universiteta. Seriya: Geologiya $=$ Proceedings of Voronezh State University. Series: Geology, 2020, No. 1, pp. 100-108. DOI: https://doi.org/10.17308/geology.2020.1/2518

Conflict of interest. The authors declare the absence of obvious and potential conflicts of interest related to the publication of this article.

\section{REFERENCES}

1. Zhavoronkin V. I., Gruzdev V. N., Faustova Yu. S. Karta summarnoj prodol'noj jelektricheskoj provodimosti osadochnogo chehla voronezhskogo kristallicheskogo massiva i ego obramlenija. [Map of the total longitudinal electrical conductivity of the sedimentary cover of the Voronezh crystalline massif and its frame]. Voprosy teorii i praktiki geologicheskoy interpretatsii geofizicheskikh poley. Materialy 45-y sessii Mezhdunarodnogo seminara im. D. G. Uspenskogo. [Questions of theory and practice of geological interpretation of geophysical fields. Materials of the 45th session of the International Seminar named after D. G. Uspensky]. Kazan, 2018, pp. 127-128. (In Russ.)

2. Gruzdev V. N. Total Conductivity of the Consolidated Part of the Earth's Crust. Vestnik Voronezhskogo gosudarstvennogo universiteta. Seriya: Geologiya $=$ Proceedings of Voronezh State University. Series: Geology, 2014, no. 2, pp. 182-185. (In Russ.)

3. Rokityansky I. I., Logvinov I. M., Maksimov V. M. Ploshchadnye magnitovariatsionnye issledovaniya $\mathrm{v}$ tsentral'noy chasti Russkoy platform. [Area magnetovariational studies in the central part of the Russian platform]. Izv. AN SSSR, Fizika Zemli. [Izv. USSR Academy of Sciences, Physics of the Earth], 1977, no. 9, pp. 107-112. (In Russ.)

4. Zhdanov M. S., Maksimov V. M., Gruzdev V. N. O rezul'tatakh magnitovariatsionnykh issledovaniy na territorii VKM i sopredel'nykh struktur tsentra Russkoy platform. [On the results of magnetovariational studies on the territory of the VKM and adjacent structures of the center of the Russian platform]. Elektromagnitnye zondirovaniya. Tez. dokl. VII Vses. shchkoly- seminara [Electromagnetic sounding. Thes. doc. VII All schools - seminar]. Moscow, 1984, p. 75. (In Russ.)

5. Gruzdev V. N. Geoelektricheskaya model' Voronezhskogo kristallicheskogo massiva. [Geoelectric model of the Voronezh crystalline massif]. Voprosy teorii $i$ praktiki geologicheskoy interpretatsii gravitatsionnykh, magnitnykh $i$ elektricheskikh poley. Tez. dokl. mezhdunarodnoy konferentsii. [Questions of theory and practice of geological interpretation of gravitational, magnetic and electric fields. Thes. doc. international conference]. Voronezh, 1996, pp. 72-73. (In Russ.)

6. Maksimov V. M., Gruzdev V. N. Ispol'zovanie metodiki Shmukera pri obrabotke i interpretatsii magnitovariatsionnykh dannykh. [Use of the Schmucker method in the processing and interpretation of magnetovariational data]. Geofizicheskie metody poiskov $i$ razvedki rudnykh i nerudnykh mestorozhdeniy. [Geophysical methods of prospecting and exploration of ore and nonore deposits]. Sverdlovsk, 1986, Issue. 12, pp. 38-44. (In Russ.)

7. Gruzdev V. N., Zhavoronkin V. I. Crustal anomalies of the deep electrical conductivity of the Voronezh crystalline massif. Vestnik Voronezhskogo gosudarstvennogo universiteta. Seriya: Geologiya $=$ Proceedings of Voronezh State University. Series: Geology, 2015, no 1, pp. 94-101. (In Russ.)

8. Muravina O. M. Density model of the earth's crust of the Voronezh crystalline massif. Vestnik Voronezhskogo gosudarstvennogo universiteta. Seriya: Geologiya = Proceedings of Voronezh State University. Series: Geology, 2016, no. 1, pp. 108-114. (In Russ.)

9. Glaznev V. N., Mints O. M., Muravina O. M. Plotnostnoe modelirovanie zemnoy kory tsentral'noy chasti VostochnoEvropeyskoy platform. [Density modeling of the earth's crust in the central part of the East European platform]. Vestnik KRAUNC. Fiz.-mat. Nauki, vol. 29, 2016, no. 1, pp. 53-63. (In Russ.)

10. Mints M. V., Glaznev V. N., Muravina O. M. The deep structure of the crust of the southeast of the Voronezh crystalline massif according to geophysical data: geodynamic evolution in the Paleoproterozoic and the modern state of the crust. Vestnik Voronezhskogo gosudarstvennogo universiteta. Seriya: Geologiya $=$ Proceedings of Voronezh State University. Series: Geology, 2017, no. 4, pp. 5-23. (In Russ.)
Груздев Владислав Николаевич - к. ф.-м. н., доцент, Воронежский государственный университет, Воронеж, Российская федерация; E- mail: grumerr@ rambler.ru; ORCID https://orcid.org/0000-0002-0654-9577

Автор прочитал и одобрил окончательный вариант рукописи.
Vladislav N. Gruzdev - PhD in Math. and Phys., associate professor, Voronezh State University, Voronezh, Russian Federation; E-mail: grumerr@ rambler.ru; ORCID https://orcid.org/0000-0002-0654-9577

Author have read and approved the final manuscript. 